OPEN ACCESS

Edited by:

Huihua Yuan,

Nantong University, China

Reviewed by:

Tao Deng,

Chongqing Medical University, China

Qiuyu Gong,

Independent Researcher, Singapore,

Singapore

*Correspondence:

Zongjiang Yu

yuzi@qibebt.ac.cn

Chao Wang

wangchao@qdu.edu.cn

Renshuai Zhang

zhangrenshuai@qdu.edu.cn

${ }^{+}$These authors have contributed equally to this work

Specialty section:

This article was submitted to Biomaterials,

a section of the journal

Frontiers in Bioengineering and

Biotechnology

Received: 20 September 2021

Accepted: 18 October 2021

Published: 03 November 2021

Citation:

Jiang $H$, Lin $Q, Y u Z$, Wang $C$ and Zhang $R$ (2021) Nanotechnologies for Reactive Oxygen Species"Turn-

On" Detection.

Front. Bioeng. Biotechnol. 9:780032.

doi: 10.3389/fbioe.2021.780032

\section{Nanotechnologies for Reactive Oxygen Species"Turn-On" Detection}

\author{
Hongfei Jiang ${ }^{1 \dagger}$, Qian Lin $^{1 \dagger}$, Zongjiang $\mathrm{Yu}^{2 *}$, Chao Wang ${ }^{1 *}$ and Renshuai Zhang ${ }^{1 *}$ \\ ${ }^{1}$ Cancer Institute, The Affiliated Hospital of Qingdao University and Qingdao Cancer Institute, Qingdao, China, ${ }^{2}$ Key Laboratory of \\ Biobased Materials, Qingdao Institute of Bioenergy and Bioprocess Technology, Chinese Academy of Sciences, Qingdao, China
}

Reactive oxygen species (ROS) encompasses a collection of complicated chemical entities characterized by individually specific biological reactivities and physicochemical properties. ROS detection is attracting tremendous attention. The reaction-based nanomaterials for ROS "turn-on" sensing represent novel and efficient tools for ROS detection. These nanomaterials have the advantages of high sensitivity, real-time sensing ability, and almost infinite contrast against background. This review focuses on appraising nanotechnologies with the ROS "turn-on" detection mechanism coupled with the ability for broad biological applications. In this review, we highlighted the weaknesses and advantages in prior sensor studies and raised some guidelines for the development of future nanoprobes.

Keywords: reactive oxygen species, ROS nanotechnology, ROS turn-on detection, detection method, sensor

\section{INTRODUCTION}

Reactive oxygen species (ROS) is the group of reactive anionic and neutral small molecules which are produced within many cell types. It mainly includes singlet oxygen $\left({ }^{1} \mathrm{O}_{2}\right)$, superoxide anion $\left(\mathrm{O}_{2}{ }^{--}\right)$, hydroxyl radical $\left({ }^{\circ} \mathrm{OH}\right)$, and hydrogen peroxide $\left(\mathrm{H}_{2} \mathrm{O}_{2}\right)$ (Yang et al., 2019). ROS has been confirmed to play a significant role in regulating numerous physiological functions of living organisms. However, ROS overproduction leads to oxidative stress and results in oxidative damage to a number of biomolecules including lipids, nucleic acids, proteins, and carbohydrates (Mattila et al., 2015), which is implicated in various diseases such as cancer, cardiovascular disease, diabetes mellitus, and aging (Valko et al., 2007; Winyard et al., 2011). Therefore, to improve the understanding of redox biology, the source and the stimulation of ROS generation, along with the consequences, we need to monitor and quantify ROS in cells, tissues, and whole organisms. Furthermore, the accurate species needs to be identified for each biological condition to fully understand redox biology.

Joint efforts have been made by chemists and biologists to monitor the locations and concentrations of these highly aggressive species with very short lifetime. Thanks to these precise ROS detection methods, remarkable progress has been witnessed in unveiling the relevant biological mechanisms and uncovering the apparently paradoxical roles of distinct ROS in human health and disease. Small molecule fluorescent probes, especially reaction-based "turn-on" fluorescent probes, are generally useful owing to their high levels of sensitivity and capability to be applied in temporal and spatial sampling for in vivo and live cell imaging (Wu et al., 2019; Wang et al., 2020; Zhang et al., 2021). Alternatively, great varieties of nanomaterials with peculiar ROS-regulating abilities have been fabricated to support ROS science in the aspects of ROS generation, depletion, transition, and detection (Zhou Z. et al., 2016); these nanotechnologies finally benefit the ROS-based therapeutic outcomes (Yang et al., 2019; Yu et al., 2020; Zhang et al., 2021; Zheng et al., 2021). Nanoparticles exhibit tunable properties in size, shape, and function that make them flexible in process and product control for a wide range of applications (He et al., 2021; Yang et al., 2021; Yin et al., 2021). Using nanoparticles 
as probes, probe vectors, and compartmentalization agents for ROS detection has become more and more popular. Rationally designed nanotechnologies for ROS "turn-on" detection (Figure 1) are expected to possess the advantages of tunable functional group control, low cellular toxicity, high levels of sensitivity, and in particular, the capability of temporal and spatial sampling for in vivo and living cell imaging (Wu et al., 2019). It is worth mentioning that the benefit of "turn-on" over "turn-off" sensors is that they have almost infinite contrast against background. In principle, nanomaterials for ROS "turn-on" detection include carbon dots, silica nanoparticles, metal-organic framework (MOF), and nanoflakes. Herein, a selection of state-of-the-art nanomaterials for "turn-on" sensing of ROS with demonstrated promising application in biological systems is reviewed. We will appraise in detail these nanotechnologies with exactly demonstrated reaction mechanisms to help researchers choose suitable nanoprobes or inspire the development of future nanotechnologies.

\section{DETECTION OF $\mathrm{H}_{2} \mathrm{O}_{2}$}

$\mathrm{H}_{2} \mathrm{O}_{2}$ is a reactive species among ROS and is closely related to various physiological processes, such as cell proliferation, apoptosis, differentiation, and other signal transmissions. Therefore, the accumulation of excessive $\mathrm{H}_{2} \mathrm{O}_{2}$ has been implicated in many diseases. Thus, it is of great significance in monitoring the concentration of $\mathrm{H}_{2} \mathrm{O}_{2}$ in a physiological environment. As $\mathrm{H}_{2} \mathrm{O}_{2}$ is the most studied species of ROS, numerous mapping tools including radiative recombination mechanism and "dark" biological processes have been developed for $\mathrm{H}_{2} \mathrm{O}_{2}$ detection ( $\mathrm{Wu}$ et al., 2019). Inspired by the successful development of boronic acidbased molecular fluorescent probes for $\mathrm{H}_{2} \mathrm{O}_{2}$ detection with a "turnon" mechanism (Bull et al., 2013), several boronic acid functionalized fluorescent nanoprobes have been designed for "turn-on" sensing of $\mathrm{H}_{2} \mathrm{O}_{2}$. This design relies on the formation of the non-fluorescence boronic acid/boronate ester, which contains an electrophilic boron center; it reacts rapidly with $\mathrm{H}_{2} \mathrm{O}_{2}$, resulting in accelerated oxidative cleavage to afford the corresponding phenol to "turn on" the fluorescence (Figure 2A). Depending on this mechanism, $\mathrm{Wu}$ and co-workers developed a fluorescence resonance energy transfer (FRET)-based ratiometric fluorescent probe for the detection of $\mathrm{H}_{2} \mathrm{O}_{2}$. This nanoparticle uses carbon dots as the energy donor and carrier. The small size $(\sim 4 \mathrm{~nm})$ and good in vivo utility of this nanoparticle may support its eventual application in clinics (limit of detection $(L O D)=0.5 \mu \mathrm{M}$ ). Higher selectivity has been achieved for the nanoparticle for the detection of $\mathrm{H}_{2} \mathrm{O}_{2}$ over other ROS and biologically relevant species (Wu et al., 2014). Zhao's group attached boronate ester to the surface of functional mesoporous silica nanoparticles (MSNPs) for "turnon" detection of $\mathrm{H}_{2} \mathrm{O}_{2}$ ( $\left.\mathrm{LOD}=3.33 \mu \mathrm{M}\right)$. Moreover, they fabricated a $\mathrm{H}_{2} \mathrm{O}_{2}$-triggered drug release system for heart failure therapy. This system exhibits the potential for different variants of heart failure models to target theranostic treatment (Tan et al., 2017). The first MOF for $\mathrm{H}_{2} \mathrm{O}_{2}$ sensing have been designed by Sk and coworkers. Different from the previous two nanoparticles, the MOF directly uses boronic acid as a functional group attached to a $\mathrm{Zr}(\mathrm{IV})$

\section{ROS responsive nanomaterials}

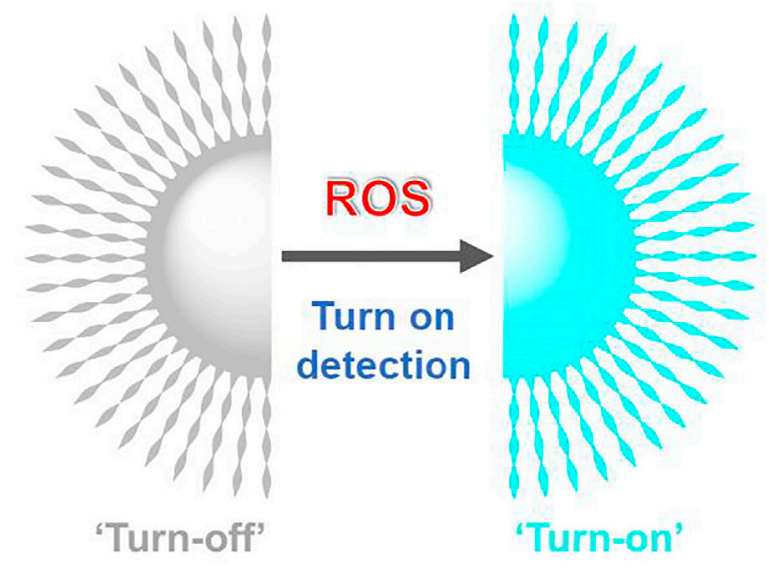

FIGURE 1 | Nanotechnologies for ROS "turn-on" detection.

MOF for "turn-on" sensing of $\mathrm{H}_{2} \mathrm{O}_{2}$ in live cells ( $\mathrm{LOD}=0.015 \mu \mathrm{M}$ ). However, it also has moderate response to some other ROS and biologically relevant species, indicating that the selectivity of this MOF material toward $\mathrm{H}_{2} \mathrm{O}_{2}$ is a major defect (Sk et al., 2018). Then a boronic acid-functionalized 3D indium MOF was fabricated for $\mathrm{H}_{2} \mathrm{O}_{2}$ detection. The MOF exhibits an improved selectivity for $\mathrm{H}_{2} \mathrm{O}_{2}$ with an LOD of $420 \mathrm{nM}$ (Jiang et al., 2021). In general, boronic acidfunctionalized nanomaterials are easier for fabrication, while boronate ester nanomaterials possess higher selectivity for $\mathrm{H}_{2} \mathrm{O}_{2}$.

The carbon dot-based fluorescence "turn-on" probe for $\mathrm{H}_{2} \mathrm{O}_{2}$ with a photo-induced electron transfer (PET) mechanism was fabricated by Zhang's group. In this nanoprobe, diphenylphosphine moiety is covalently attached to the surface of the carbon dot; they serve as the PET donor and acceptor, respectively. Subsequently, $\mathrm{H}_{2} \mathrm{O}_{2}$ can selectively oxidize the diphenylphosphine to produce the target oxide and prevent the PET mechanism; then the fluorescence will "turn on" (Figure 2B). The nanoprobe has a fast response to $\mathrm{H}_{2} \mathrm{O}_{2}$ with a LOD of $84 \mathrm{nM}$ (Lan et al., 2015). Peroxalate-functionalized carbon nanodots are novel near-infrared chemiluminescent nanomaterials for $\mathrm{H}_{2} \mathrm{O}_{2}$ detection $(\mathrm{LOD}=5 \mathrm{nM})$. Nanointegration of near-infrared carbon nanodots and peroxalate (P-CDs) with amphiphilic triblock copolymer as bridge can serve as "turn-on" sensors for the detection and imaging of $\mathrm{H}_{2} \mathrm{O}_{2}$ (Figure 2C). The high efficiency and large penetration depth of near-infrared photons of P-CDs make this strategy a good choice for bioimaging of $\mathrm{H}_{2} \mathrm{O}_{2}$ in vitro and in vivo (Shen et al., 2020).

Ag-based nanomaterials have broad application for $\mathrm{H}_{2} \mathrm{O}_{2}$ detection. In these designs, Ag materials normally act as a shell and serve as efficient quenchers, while $\mathrm{H}_{2} \mathrm{O}_{2}$ can prevent the $\mathrm{Ag}$ material-mediated quenching mechanism and fulfill the "turn-on" detection of $\mathrm{H}_{2} \mathrm{O}_{2}$ (Figure 2D). Chu's group utilized DNAtemplated Ag nanoparticles (DNA-AgNPs) coupled with NaYF4:Yb/Tm@NaYF4 shell upconversion nanoparticles (UCNPs) for the detection of $\mathrm{H}_{2} \mathrm{O}_{2}$ (LOD $\left.=1.08 \mu \mathrm{M}\right)$, in which, UCNPs and DNA-AgNPs serve as donors and 
A

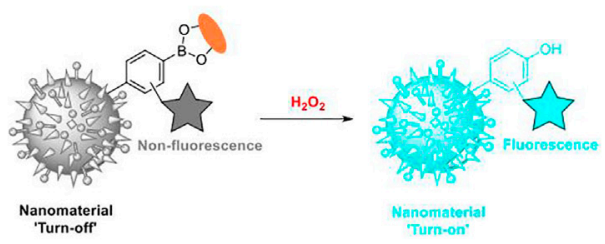

C

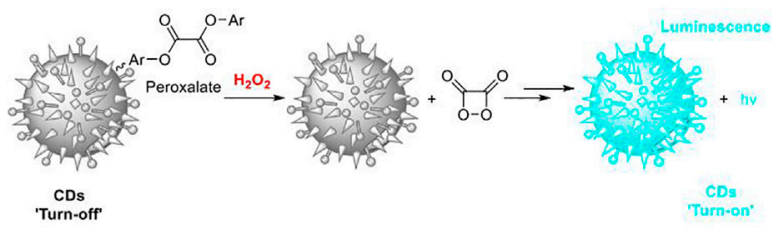

E

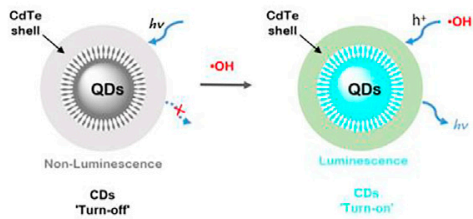

G

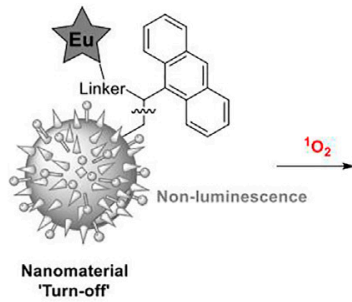

I

$\mathbf{F}$

H

$\mathbf{J}$

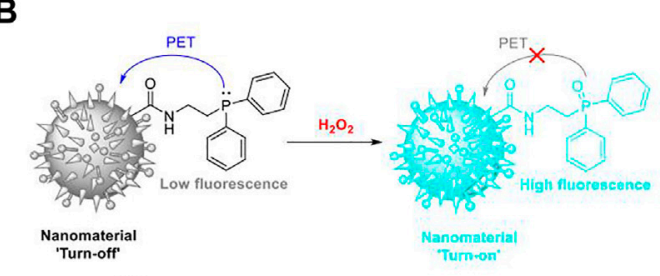

D)
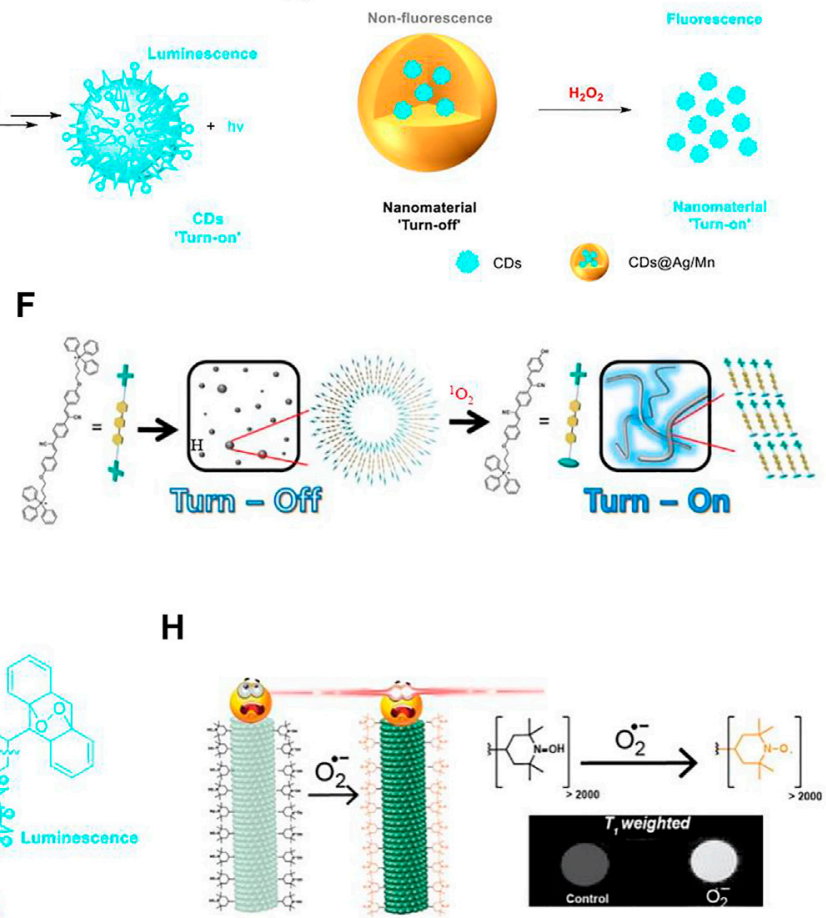

Fluorescence Non-fluorescence Fluorescence
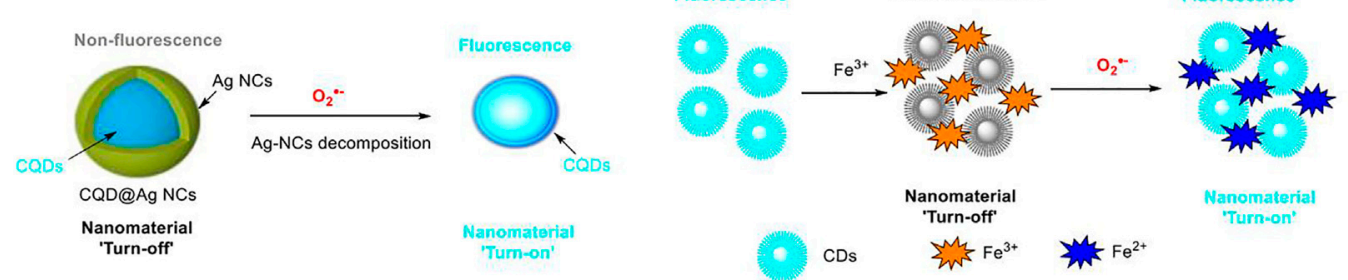

L

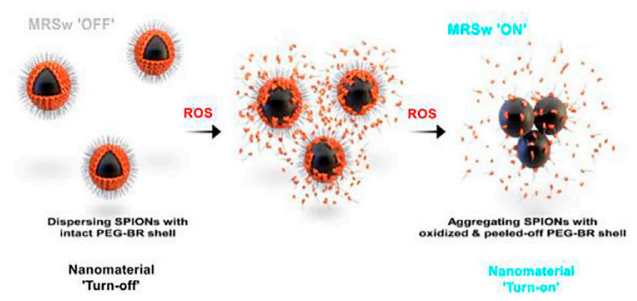

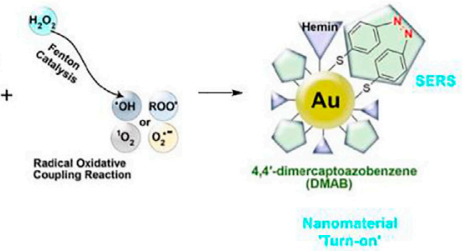

FIGURE 2 | Nanotechnologies for ROS detection with a "turn-on" mechanism. (A) Boronic acid/boronate ester-based nanomaterials for $\mathrm{H}_{2} \mathrm{O}_{2}$ "turn-on" detection; (B) carbon dot-based fluorescence "turn-on" probe for $\mathrm{H}_{2} \mathrm{O}_{2}$ detection with a PET mechanism; (C) peroxalate-functionalized carbon nanodots as near-infrared chemiluminescent nanomaterial for $\mathrm{H}_{2} \mathrm{O}_{2}$ "turn-on" detection; (D) Ag -and Mn-based nanomaterials for $\mathrm{H}_{2} \mathrm{O}_{2}$ "turn-on" detection; (E) semiconductor quantum dots as "turn-on" luminescent probes for real-time detection of $\bullet \mathrm{OH}$; (F) triphenylphosphonium-based self-assembled nanomaterial for ${ }^{1} \mathrm{O}_{2}$ "turn-on" detection; (G) "turnon" detection of ${ }^{1} \mathrm{O}_{2}$ using a nanostructured porous silicon microcavity through photonic luminescence enhancement strategy; (H) metal-free magnetic resonance imaging (MRI) tool for $\mathrm{O}_{2}{ }^{\bullet}$ " "turn-on" detection (copyright 2018 American Chemical Society); (I) schematic illustrations of fluorescence "turn-on" detection of O2 ${ }^{\bullet}$ - based on CQD@Ag NCs (copyright 2017 Springer); (J) illustration of CDs-Fe ${ }^{3+}$ for the detection of $\mathrm{O}_{2}{ }^{-}$; (K) PEG-BR@SPIONs as the biosensor with a magnetic relaxation switching-based mechanism for ROS "turn-on" detection; (L) Au-PATP-Hemin nanoprobe for ROS "turn-on" detection (copyright 2018 American Chemical Society). 
quenchers, respectively. This design results in luminescence quenching of UCNPs using DNA-AgNPs by luminescence resonance energy transfer (LRET). Upon $\mathrm{H}_{2} \mathrm{O}_{2}$ introduction, AgNPs can be converted to $\mathrm{Ag}^{+}$, leading to the inhibition of the LRET process and inducing the recovery of upconversion luminescence ( $\mathrm{Wu}$ et al., 2016). In this way, graphene quantum dots (QDs) adopted with the silver shell (GQD@Ag, LOD = $2 \mu \mathrm{M}$ ) (Kong et al., 2017), nitrogen-doped carbon QDs coated with silver nanoparticles (N-CQD/AgNPs, LOD $=4.7 \mu \mathrm{M}$ ) (Walekar et al., 2017), and a novel nanocluster-mediated chemical information processing system (CIPS, LOD information unavailable) (Zhao et al., 2018) have been designed and applied in selective $\mathrm{H}_{2} \mathrm{O}_{2}$ sensing with a "turn-on" mechanism.

Alternatively, the Mn-mediated nanotechnologies share a similar mechanism with Ag-mediated nanomaterials for $\mathrm{H}_{2} \mathrm{O}_{2}$ "turn-on" detection but have higher selectivity (Figure 2D). In this process, $\mathrm{MnO}_{2}$ nanosheets serve as a quencher but can be oxidized by $\mathrm{H}_{2} \mathrm{O}_{2}$ to fulfill the "turn-on" sensing of $\mathrm{H}_{2} \mathrm{O}_{2}$. Depending on this design, Yuan and co-workers fabricated manganese dioxide $\left(\mathrm{MnO}_{2}\right)$-nanosheet-modified UCNPs for rapid detection of $\mathrm{H}_{2} \mathrm{O}_{2}(\mathrm{LOD}=0.9 \mu \mathrm{M})$. The $\mathrm{MnO}_{2}$ nanosheets on the surface of UCNPs serve as the quencher. Fluorescence of UCNPs will be recovered after the addition of $\mathrm{H}_{2} \mathrm{O}_{2}$, which can reduce $\mathrm{MnO}_{2}$ to $\mathrm{Mn}^{2+}$ and destroy the structure of the $\mathrm{MnO}_{2}$ quencher (Yuan et al., 2015). Following this design, Lei and Liu's group developed a carbon dot $-\mathrm{MnO}_{2}$ probe $(\mathrm{LOD}=$ $0.87 \mu \mathrm{M})$ and a three-in-one stimulus-responsive nanoplatform (Au@MnO2@Raman reporter, LOD =6-7 $\mu \mathrm{M}$ ), respectively, for $\mathrm{H}_{2} \mathrm{O}_{2}$ sensing with relatively improved selectivity or sensitivity (Ning et al., 2020; Zhang et al., 2020). While the Ag and Mnmediated nanomaterials are utilized for $\mathrm{H}_{2} \mathrm{O}_{2}$ sensing in solutions, the biocompatibility of such structures is still questionable.

\section{DETECTION OF HYDROXYL RADICAL ('OH)}

• $\mathrm{OH}$, the result of the homolytic cleavage of water $\left(\mathrm{H}_{2} \mathrm{O} \rightarrow{ }^{\bullet} \mathrm{OH}+\right.$ $\left.{ }^{\bullet} \mathrm{H}\right)$, is the most deleterious and reactive species of ROS. The general reactivity of the main ROS in biological systems decreases in the order of ${ }^{\bullet} \mathrm{OH}>{ }^{1} \mathrm{O}_{2}>\mathrm{H}_{2} \mathrm{O}_{2}>\mathrm{O}_{2}{ }^{\bullet-}$ (Mattila et al., 2015). ${ }^{\bullet} \mathrm{OH}$ can destroy a number of biomolecules including proteins, lipids, and DNA, so as to induce numerous oxidative stress-related diseases. However, at present time, the detailed function of ${ }^{\bullet} \mathrm{OH}$ has seldom been demonstrated owing to the extremely high reactivity and short lifetime (Bai et al., 2019). Therefore, real-time sensing of ${ }^{\bullet} \mathrm{OH}$ in biological samples is of great importance. The use of semiconductor QDs as "turn-on" luminescent probes for real-time detection of ${ }^{\circ} \mathrm{OH}$ has been developed (LOD $=0.3 \mu \mathrm{M}$ ) (Figure 2E). In this design, metal citrate complexes are adopted on the surfaces of QDs and can act as electron donors, injecting electrons into the lowest unoccupied molecular orbital (LUMO) of the QDs. Interestingly, only ${ }^{\bullet} \mathrm{OH}$ can inject holes into the highest occupied molecular orbital (HOMO) of the QDs. Consequently, the produced electron-hole pairs could emit strong luminescence through electron-hole recombination. This nanotechnology is demonstrated to have an application in detecting the endogenous release of ${ }^{\bullet} \mathrm{OH}$ in living cells (Zhou W. et al., 2016).
Alternatively, Yu's group fabricated a polyhedral-AuPd nanoparticle-based dual-mode cytosensor (PH-AuPd NPs, LOD information unavailable) with a "turn-on"-enabled signal for ${ }^{\bullet} \mathrm{OH}$ sensing. In this strategy, tetramethylbenzidine (TMB) acting as a functional group on the cytosensor is oxidized to ox TMB, a colored product, and can be monitored through colorimetric analysis. Coupled with a rational design, the nanotechnology has been constructed as a convenient method for the sensitive detection of MCF-7 cells (LOD $=20$ cells $\mathrm{ml}^{-1}$ ) (Wang H. et al., 2018).

\section{DETECTION OF SINGLET OXYGEN}

${ }^{1} \mathrm{O}_{2}$ has raised vital interest recently as a result of its significance in both chemical and biological systems. ${ }^{1} \mathrm{O}_{2}$ is the lowest excited electronic state of molecular oxygen but is recognized to be highly reactive. Studies have demonstrated that ${ }^{1} \mathrm{O}_{2}$ is highly toxic and destroys key biological molecules including proteins, DNA, and unsaturated lipids. Depending on a triphenylphosphonium derivative, the self-assembled nanomaterial has been fabricated for ${ }^{1} \mathrm{O}_{2}$ "turn-on" detection (LOD $=33-56 \mu \mathrm{M}$ ) (Figure 2F). However, these nanoparticles are responsive to both ${ }^{1} \mathrm{O}_{2}$ and $\mathrm{ClO}^{-}$(Choi et al., 2018). A strategy for "turn-on" detection of ${ }^{1} \mathrm{O}_{2}$ using a nanostructured porous silicon microcavity ( $\mathrm{pSiMC}, \mathrm{LOD}$ $=37 \mathrm{nM}$ ) through photonic luminescence enhancements has been developed (Figure 2G). The pSiMC is modified with an $\mathrm{Eu}$ (III)-linker-anthracene complex. In the presence of ${ }^{1} \mathrm{O}_{2}$, the formation of an endoperoxide in the 9,10 position of anthracene is confirmed. Changes in the anthracene moiety can result in changes to the emission of the $\mathrm{Eu}$ (III) ion so as to induce these nanoprobes to become luminescent (Jenie et al., 2017). Alternatively, a technique for electrical detection of ${ }^{1} \mathrm{O}_{2}$ on the surface of silver nanoparticle film has been fabricated by Knoblauch and co-workers. Singlet oxygen sensor green (SOSG, LOD information unavailable) in this system functions as a crucial moiety for the fluorescence "turn-on" sensing process. The presence of ${ }^{1} \mathrm{O}_{2}$ in this system can result in change in the SOSG fluorescence quantum yield, which permits a stronger energy transfer from the SOSG probe to a proximal silver nanoparticle island film located in the nearelectric field of the probe. This induces an increase in the target electric current flow, allowing for the sensing of the ${ }^{1} \mathrm{O}_{2}$ (Knoblauch et al., 2020).

\section{DETECTION OF SUPEROXIDE $\left(\mathrm{O}_{2}{ }^{--}\right)$}

$\mathrm{O}_{2}{ }^{\bullet-}$ is a by-product of ATP generation processes of the human body microenvironment, which plays a significant role in regulating biochemistry and organic pathology. Furthermore, exposure to excess $\mathrm{O}_{2}{ }^{\bullet-}$ would oxidize organisms, biological membranes, and tissues and cause diseases such as hepatitis, cancer, and diabetes (Gorrini et al., 2013). Nanomaterials such as carbon dots (Liang et al., 2020; Yue et al., 2021), MOF (Das et al., 2019), and tobacco mosaic virus (TMV) nanoparticles (Dharmarwardana et al., 2018) have been fabricated as selective sensors for "turn-on" sensing of $\mathrm{O}_{2}{ }^{\bullet-}$. Silver nanoparticle (Ag NP)-coated carbon quantum dot (CQD) 
core-shell-structured nanocomposites (CQD@Ag NCs) have been developed for fluorescent sensing of intracellular $\mathrm{O}_{2}{ }^{--}(\mathrm{LOD}=$ $0.3 \mu \mathrm{M}$ ) (Figure 2I). In CQD@Ag NCs, CQDs display a potent blue fluorescence; however, the fluorescence is quenched by Ag NPs. In the presence of $\mathrm{O}_{2}{ }^{--}$, Ag NPs are oxide-etched, and the fluorescence of CQDs is recovered (Liang et al., 2020). Yue and co-workers fabricated similar carbon dots for $\mathrm{O}_{2}{ }^{\bullet-}$ sensing while the quencher is $\mathrm{Fe}^{3+}(\mathrm{LOD}=25 \mathrm{pM})$. The addition of $\mathrm{O}_{2}^{--}$can convert $\mathrm{Fe}^{3+}$ to $\mathrm{Fe}^{2+}$ and recover the fluorescence of carbon dots (Yue et al., 2021) (Figure 2J). It is reported that CQD@Ag NCs are successfully utilized in the imaging of $\mathrm{O}_{2}{ }^{--}$in MCF-7 cells; however, the biocompatibility of Liang's carbon dots is questionable. The Gassensmith group managed to functionalize the surface of TMV (LOD information unavailable) nanoparticles with 4-hydroxytetramethylpiperidine at the protein tyrosine residue. The nanoparticles function as a metal-free magnetic resonance imaging (MRI) tool for $\mathrm{O}_{2}{ }^{\bullet-}$ monitoring (Figure 2H). The mechanism of this strategy for $\mathrm{O}_{2}^{--}$"turn-on" detection is that 4-hydroxytetramethylpiperidine can be oxidized to TEMPO, which has a different $T_{1}$-weighted imaging. TMV nanoparticles can selectively respond to $\mathrm{O}_{2}{ }^{\bullet-}$ without being affected by $\mathrm{H}_{2} \mathrm{O}_{2}$ and $\mathrm{O}_{2}$; however, no available data show whether other species of ROS, for example, the more reactive ${ }^{\circ} \mathrm{OH}$, can react with TMV nanoparticles (Dharmarwardana et al., 2018). However, a MOF material of the $\mathrm{UiO}$ family called $\mathrm{Zr}-\mathrm{UiO}-66-\mathrm{NH}-\mathrm{CH} 2-\mathrm{Py}$ has been fabricated with a clear selectivity toward $\mathrm{O}_{2}{ }^{--}$over other $\mathrm{ROS}(\mathrm{LOD}=0.21 \mu \mathrm{M})$. Enhancement of the fluorescence response of the MOF upon stepwise addition of $\mathrm{O}_{2}{ }^{\bullet-}$ has been recorded. The mechanism of the fluorescence "turn-on" procedure is recognized as follows: the structural collapse of the MOF in the presence of $\mathrm{O}_{2}{ }^{\bullet-}$ can result in the release of the linker (2-((pyridin-4-ylmethyl)amino)terephthalic acid) with the enhancement of the fluorescence intensity of the system (Das et al., 2019).

\section{DETECTION OF COMBINED SPECIES OF ROS}

In some conditions, the evaluation of cellular or system total ROS provides helpful information on cell proliferation, metabolism, and tumor detection. Distinct from the design of nanotechnologies for selective sensing of specific species of ROS, these nanomaterials can detect the combined species of ROS by one platform. Several nanomaterials, including PEGylated bilirubin-coated superparamagnetic iron oxide nanoparticles (PEG-BR@SPIONs) (Lee et al., 2020), UCNPs-MoS ${ }_{2}$ nanoflakes (Wang F. et al., 2018), multifunctional theranostic nanoprobes (Au-Ag-HM) (Wang et al., 2021), para-aminothiophenol and hemin-decorated gold (Au-PATP-Hemin) nanoprobes (Cui et al., 2018), cyclotriphosphazene-doped graphene quantum dots (C-GQDs) (Xu et al., 2020), ROS-responsive microgel (Liu et al., 2018), and the ionic nanoparticles in a hydrogel microparticle (Liu et al., 2018), are designed under this context. Therein, PEGBR@SPIONs and Au-PATP-Hemin nanoprobe are promising tools for "turn-on" detection of ROS with demonstrated mechanisms and have potentialities in biological applications.
PEG-BR@SPIONs as a biosensor with a magnetic relaxation switching-based mechanism have been employed for wholeblood ROS sensing (LOD $=30-50 \mu \mathrm{M}$ ) (Figure $2 \mathrm{~K}$ ). The "turnon" mechanism is actualized by the change of magnetic relaxation signal upon exposure to ROS. Furthermore, these ROS-responsive PEG-BR@SPIONs are utilized in a sepsis-mimetic clinical setting to directly monitor the total concentration of ROS in the blood samples through an explicit change in $T_{2}$ magnetic relaxation signals and a "turn-on" signal of fluorescence. The design of a Au-PATP-Hemin nanoprobe is principled upon the discovery that PATP can react with ROS through a radical oxidative coupling mechanism to form 4, $4^{\prime}$-dimercaptoazobenzene (DMAB), which can elicit potent characteristic surface-enhanced Raman scattering (SERS) signals at $1,142,1,386$, and $1,432 \mathrm{~cm}^{-1}$ and directly enable the detection of ROS through a hemin-catalyzed Fenton reaction $(\mathrm{LOD}=26 \mathrm{pM})$ (Figure 2L). Simultaneous detection of five ROS species ( ${ }^{\bullet} \mathrm{OH}, \mathrm{ROO}^{\bullet}, \mathrm{O}_{2}{ }^{\bullet-},{ }^{1} \mathrm{O}_{2}$, and $\mathrm{H}_{2} \mathrm{O}_{2}$ ) has been realized by the Au-PATP-Hemin nanoprobe. In two typical ROS-elevated mice models of allergic dermatitis and tumors, the Au-PATP-Hemin nanoprobe performed well in monitoring inflammation progression and tumor development in a sensitive and quantitative manner.

\section{CONCLUSION}

In this short review, nanomaterials with the ability for ROS "turnon" detection have been deciphered from the aspects of both nanotechnology and chemical reaction mechanisms. In general, "turn-on" nanotechnologies are powerful tools with a low detection limit, real-time sensing ability, and almost infinite contrast against background. Future studies for the design of ROS "turn-on" detection nanomaterials should make efforts to improve selectivity, detection limit, and biocompatibility. Another consideration is the accessibility of these nanomaterials. Reagents for the nanomaterial fabrication are commercially available or can be prepared in simple synthetic steps from commercially available building blocks, which will be greatly in vogue. Continuous efforts are poised to develop more powerful nanotechnologies in this promising field to shed light on critical information of ROS in biological systems.

\section{AUTHOR CONTRIBUTIONS}

$\mathrm{RZ}$, CW, and ZY conceived the conceptualization of the manuscript. All authors contributed to the discussion and composition of the content and helped write the manuscript.

\section{FUNDING}

This study was supported by the Shandong Provincial Natural Science Foundation, China (No. ZR2020QC081, HJ), and Youth Innovation Team Talent Introduction Program of Shandong Province (20190164, RZ and HJ). 


\section{REFERENCES}

Bai, X., Ng, K. K.-H., Hu, J. J., Ye, S., and Yang, D. (2019). Small-molecule-based Fluorescent Sensors for Selective Detection of Reactive Oxygen Species in Biological Systems. Annu. Rev. Biochem. 88, 605-633. doi:10.1146/annurevbiochem-013118-111754

Bull, S. D., Davidson, M. G., van den Elsen, J. M. H., Fossey, J. S., Jenkins, A. T. A., Jiang, Y.-B., et al. (2013). Exploiting the Reversible Covalent Bonding of Boronic Acids: Recognition, Sensing, and Assembly. Acc. Chem. Res. 46, 312-326. doi:10.1021/ar300130w

Choi, W., Lim, N., Choi, H., Seo, M., Ahn, J., and Jung, J. (2018). Self-assembled Triphenylphosphonium-Conjugated Dicyanostilbene Nanoparticles and Their Fluorescence Probes for Reactive Oxygen Species. Nanomaterials 8, 1034. doi:10.3390/nano8121034

Cui, K., Fan, C., Chen, G., Qiu, Y., Li, M., Lin, M., et al. (2018). Paraaminothiophenol Radical Reaction-Functionalized Gold Nanoprobe for One-To-All Detection of Five Reactive Oxygen Species In Vivo. Anal. Chem. 90, 12137-12144. doi:10.1021/acs.analchem.8b03116

Das, A., Anbu, N., Sk, M., Dhakshinamoorthy, A., and Biswas, S. (2019). A Functionalized Uio-66 Mof for Turn-On Fluorescence Sensing of Superoxide in Water and Efficient Catalysis for Knoevenagel Condensation. Dalton Trans. 48, 17371-17380. doi:10.1039/c9dt03638e

Dharmarwardana, M., Martins, A. F., Chen, Z., Palacios, P. M., Nowak, C. M., Welch, R. P., et al. (2018). Nitroxyl Modified Tobacco Mosaic Virus as a Metalfree High-Relaxivity MRI and EPR Active Superoxide Sensor. Mol. Pharmaceutics 15, 2973-2983. doi:10.1021/acs.molpharmaceut.8b00262

Gorrini, C., Harris, I. S., and Mak, T. W. (2013). Modulation of Oxidative Stress as an Anticancer Strategy. Nat. Rev. Drug Discov. 12, 931-947. doi:10.1038/ nrd4002

He, Y., Zhao, W., Dong, Z., Ji, Y., Li, M., Hao, Y., et al. (2021). A Biodegradable Antibacterial Alginate/carboxymethyl Chitosan/kangfuxin Sponges for Promoting Blood Coagulation and Full-Thickness Wound Healing. Int. J. Biol. Macromol. 167, 182-192. doi:10.1016/j.ijbiomac.2020.11.168

Jenie, S. N. A., Plush, S. E., and Voelcker, N. H. (2017). Singlet Oxygen Detection on a Nanostructured Porous Silicon Thin Film via Photonic Luminescence Enhancements. Langmuir 33, 8606-8613. doi:10.1021/acs.langmuir.7b00522

Jiang, X., Fan, R., Zhou, X., Zhu, K., Sun, T., Zheng, X., et al. (2021). Mixed Functionalization Strategy on Indium-Organic Framework for Multiple Ion Detection and H2O2 Turn-On Sensing. Dalton Trans. 50, 7554-7562. doi:10.1039/d1dt00889g

Knoblauch, R., Moskowitz, J., Hawkins, E., and Geddes, C. D. (2020). Fluorophoreinduced Plasmonic Current: Generation-Based Detection of Singlet Oxygen. ACS Sens. 5, 1223-1229. doi:10.1021/acssensors.0c00377

Kong, R.-M., Yang, A., Wang, Q., Wang, Y., Ma, L., and Qu, F. (2017). Uricase Based Fluorometric Determination of Uric Acid Based on the Use of Graphene Quantum Dot@silver Core-Shell Nanocomposites. Microchim. Acta 185, 63. doi:10.1007/s00604-017-2614-4

Lan, M., Di, Y., Zhu, X., Ng, T.-W., Xia, J., Liu, W., et al. (2015). A Carbon DotBased Fluorescence Turn-On Sensor for Hydrogen Peroxide with a PhotoInduced Electron Transfer Mechanism. Chem. Commun. 51, 15574-15577. doi:10.1039/c5cc05835j

Lee, D. Y., Kang, S., Lee, Y., Kim, J. Y., Yoo, D., Jung, W., et al. (2020). Pegylated Bilirubin-Coated Iron Oxide Nanoparticles as a Biosensor for Magnetic Relaxation Switching-Based Ros Detection in Whole Blood. Theranostics 10, 1997-2007. doi:10.7150/thno.39662

Liang, H., Liu, H., Tian, B., Ma, R., and Wang, Y. (2020). Carbon Quantum Dot@ silver Nanocomposite-Based Fluorescent Imaging of Intracellular Superoxide Anion. Microchim. Acta 187, 484. doi:10.1007/s00604-020-04359-8

Liu, Y., Wang, Y.-M., Sedano, S., Jiang, Q., Duan, Y., Shen, W., et al. (2018). Encapsulation of Ionic Nanoparticles Produces Reactive Oxygen Species (Ros)responsive Microgel Useful for Molecular Detection. Chem. Commun. 54, 4329-4332. doi:10.1039/c8cc01432a

Mattila, H., Khorobrykh, S., Havurinne, V., and Tyystjärvi, E. (2015). Reactive Oxygen Species: Reactions and Detection from Photosynthetic Tissues. J. Photochem. Photobiol. B: Biol. 152, 176-214. doi:10.1016/ j.jphotobiol.2015.10.001
Ning, K., Xiang, G., Wang, C., Huang, F., Liu, J., Zhang, L., et al. (2020). 'Turnon' Fluorescence Sensing of Hydrogen Peroxide in marine Food Samples Using a Carbon Dots- $\mathrm{MnO}_{2}$ Probe. Luminescence 35, 897-902. doi:10.1002/ bio. 3799

Shen, C. L., Lou, Q., Zang, J. H., Liu, K. K., Qu, S. N., Dong, L., et al. (2020). NearInfrared Chemiluminescent Carbon Nanodots and Their Application in Reactive Oxygen Species Bioimaging. Adv. Sci. 7, 1903525. doi:10.1002/ advs.201903525

Sk, M., Banesh, S., Trivedi, V., and Biswas, S. (2018). Selective and Sensitive Sensing of Hydrogen Peroxide by a Boronic Acid Functionalized Metal-Organic Framework and its Application in Live-Cell Imaging. Inorg. Chem. 57, 14574-14581. doi:10.1021/acs.inorgchem.8b02240

Tan, S. Y., Teh, C., Ang, C. Y., Li, M., Li, P., Korzh, V., et al. (2017). Responsive Mesoporous Silica Nanoparticles for Sensing of Hydrogen Peroxide and Simultaneous Treatment toward Heart Failure. Nanoscale 9, 2253-2261. doi:10.1039/c6nr08869d

Valko, M., Leibfritz, D., Moncol, J., Cronin, M. T. D., Mazur, M., and Telser, J. (2007). Free Radicals and Antioxidants in normal Physiological Functions and Human Disease. Int. J. Biochem. Cel Biol. 39, 44-84. doi:10.1016/ j.biocel.2006.07.001

Walekar, L. S., Hu, P., Liao, F., Guo, X., and Long, M. (2017). Turn-on Fluorometric and Colorimetric Probe for Hydrogen Peroxide Based on the In-Situ Formation of Silver Ions from a Composite Made from N-Doped Carbon Quantum Dots and Silver Nanoparticles. Microchim. Acta 185, 31. doi:10.1007/s00604-0172545-0

Wang, F., Qu, X., Liu, D., Ding, C., Zhang, C., and Xian, Y. (2018a). Upconversion Nanoparticles-Mos2 Nanoassembly as a Fluorescent Turn-On Probe for Bioimaging of Reactive Oxygen Species in Living Cells and Zebrafish. Sens. Actuators B: Chem. 274, 180-187. doi:10.1016/j.snb.2018.07.125

Wang, H., Zhou, C., Sun, X., Jian, Y., Kong, Q., Cui, K., et al. (2018b). PolyhedralAuPd Nanoparticles-Based Dual-Mode Cytosensor with Turn on Enable Signal for Highly Sensitive Cell Evalution on Lab-On-Paper Device. Biosens. Bioelectron. 117, 651-658. doi:10.1016/j.bios.2018.07.004

Wang, K., Zhang, F., Wei, Y., Wei, W., Jiang, L., Liu, Z., et al. (2021). In Situ imaging of Cellular Reactive Oxygen Species and Caspase-3 Activity Using a Multifunctional Theranostic Probe for Cancer Diagnosis and Therapy. Anal. Chem. 93, 7870-7878. doi:10.1021/acs.analchem.1c00385

Wang, K., Ma, W., Xu, Y., Liu, X., Chen, G., Yu, M., et al. (2020). Design of a Novel Mitochondria Targetable Turn-On Fluorescence Probe for Hydrogen Peroxide and its Two-Photon Bioimaging Applications. Chin. Chem. Lett. 31, 3149-3152. doi:10.1016/j.cclet.2020.08.039

Winyard, P. G., Ryan, B., Eggleton, P., Nissim, A., Taylor, E., Lo Faro, M. L., et al. (2011). Measurement and Meaning of Markers of Reactive Species of Oxygen, Nitrogen and Sulfur in Healthy Human Subjects and Patients with Inflammatory Joint Disease. Biochem. Soc. Trans. 39, 1226-1232. doi:10.1042/bst0391226

Wu, G., Zeng, F., Yu, C., Wu, S., and Li, W. (2014). A Ratiometric Fluorescent Nanoprobe for $\mathrm{H} 2 \mathrm{O} 2$ sensing and In Vivo Detection of Drug-Induced Oxidative Damage to the Digestive System. J. Mater. Chem. B 2, 8528-8537. doi:10.1039/ c4tb01432d

Wu, S., Kong, X.-J., Cen, Y., Yuan, J., Yu, R.-Q., and Chu, X. (2016). Fabrication of a LRET-Based Upconverting Hybrid Nanocomposite for Turn-On Sensing of H2O2and Glucose. Nanoscale 8, 8939-8946. doi:10.1039/c6nr00470a

Wu, L., Sedgwick, A. C., Sun, X., Bull, S. D., He, X.-P., and James, T. D. (2019). Reaction-based Fluorescent Probes for the Detection and Imaging of Reactive Oxygen, Nitrogen, and Sulfur Species. Acc. Chem. Res. 52, 2582-2597. doi:10.1021/acs.accounts.9b00302

Xu, A., He, P., Ye, C., Liu, Z., Gu, B., Gao, B., et al. (2020). Polarizing Graphene Quantum Dots toward Long-Acting Intracellular Reactive Oxygen Species Evaluation and Tumor Detection. ACS Appl. Mater. Inter. 12, 10781-10790. doi:10.1021/acsami.9b20434

Yang, B., Chen, Y., and Shi, J. (2019). Reactive Oxygen Species (Ros)-Based Nanomedicine. Chem. Rev. 119, 4881-4985. doi:10.1021/acs.chemrev.8b00626

Yang, L., Pijuan-Galito, S., Rho, H. S., Vasilevich, A. S., Eren, A. D., Ge, L., et al. (2021). High-throughput Methods in the Discovery and Study of Biomaterials and Materiobiology. Chem. Rev. 121, 4561-4677. doi:10.1021/ acs.chemrev.0c00752 
Yin, X., Hao, Y., Lu, Y., Zhang, D., Zhao, Y., Mei, L., et al. (2021). BioMultifunctional Hydrogel Patches for Repairing Full-Thickness Abdominal wall Defect. Adv. Funct. Mater. 31, 2105614. doi:10.1002/adfm.202105614

Yu, Z., Li, Q., Wang, J., Yu, Y., Wang, Y., Zhou, Q., et al. (2020). Reactive Oxygen Species-Related Nanoparticle Toxicity in the Biomedical Field. Nanoscale Res. Lett. 15, 115. doi:10.1186/s11671-020-03344-7

Yuan, J., Cen, Y., Kong, X.-J., Wu, S., Liu, C.-L., Yu, R.-Q., et al. (2015). Mno2nanosheet-modified Upconversion Nanosystem for Sensitive Turn-On Fluorescence Detection of $\mathrm{H} 2 \mathrm{O} 2$ and Glucose in Blood. ACS Appl. Mater. Inter. 7, 10548-10555. doi:10.1021/acsami.5b02188

Yue, J., Peng, J., Yu, L., Sun, M., Sun, Z., Tan, H., et al. (2021). Superoxide Anion Turns on the Fluorescence of Carbon Dots-Ferric Complex for Sensing. Microchemical J. 168, 106412. doi:10.1016/j.microc.2021.106412

Zhang, C., Liu, X., Xu, Z., and Liu, D. (2020). Multichannel Stimulus-Responsive Nanoprobes for $\mathrm{H} 2 \mathrm{O} 2$ Sensing in Diverse Biological Milieus. Anal. Chem. 92, 12639-12646. doi:10.1021/acs.analchem.0c02769

Zhang, W., Chen, L., Xiong, Y., Panayi, A. C., Abududilibaier, A., Hu, Y., et al. (2021). Antioxidant Therapy and Antioxidant-Related Bionanomaterials in Diabetic Wound Healing. Front. Bioeng. Biotechnol. 9, 707479. doi:10.3389/fbioe.2021.707479

Zhao, Y., Liu, H., Jiang, Y., Song, S., Zhao, Y., Zhang, C., et al. (2018). Detection of Various Biomarkers and Enzymes via a Nanocluster-Based Fluorescence Turn-On Sensing Platform. Anal. Chem. 90, 14578-14585. doi:10.1021/acs.analchem.8b04691

Zheng, W., Zhou, Q., and Yuan, C. (2021). Nanoparticles for Oral Cancer Diagnosis and Therapy. Bioinorg Chem. Appl. 2021, 9977131. doi:10.1155/ 2021/9977131
Zhou, W., Cao, Y., Sui, D., and Lu, C. (2016a). Turn-on Luminescent Probes for the Real-Time Monitoring of Endogenous Hydroxyl Radicals in Living Cells. Angew. Chem. Int. Ed. 55, 4236-4241. doi:10.1002/ anie. 201511868

Zhou, Z., Song, J., Nie, L., and Chen, X. (2016b). Reactive Oxygen Species Generating Systems Meeting Challenges of Photodynamic Cancer Therapy. Chem. Soc. Rev. 45, 6597-6626. doi:10.1039/c6cs00271d

Conflict of Interest: The authors declare that the research was conducted in the absence of any commercial or financial relationships that could be construed as a potential conflict of interest.

Publisher's Note: All claims expressed in this article are solely those of the authors and do not necessarily represent those of their affiliated organizations, or those of the publisher, the editors and the reviewers. Any product that may be evaluated in this article, or claim that may be made by its manufacturer, is not guaranteed or endorsed by the publisher.

Copyright (c) 2021 Jiang, Lin, Yu, Wang and Zhang. This is an open-access article distributed under the terms of the Creative Commons Attribution License (CC BY). The use, distribution or reproduction in other forums is permitted, provided the original author(s) and the copyright owner(s) are credited and that the original publication in this journal is cited, in accordance with accepted academic practice. No use, distribution or reproduction is permitted which does not comply with these terms. 